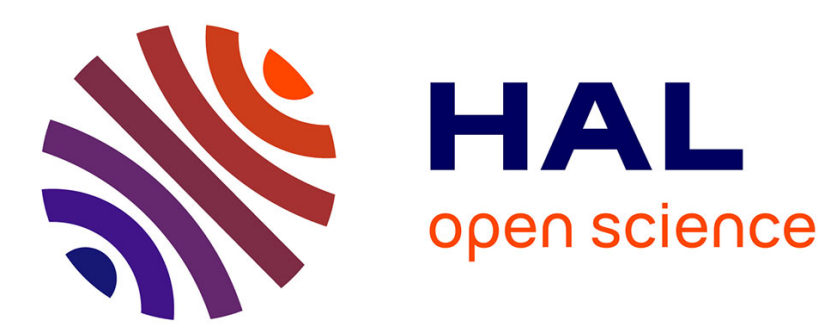

\title{
La domination mayorale - Analyser l'exercice du pouvoir des maires comme une société de cour
}

Virginie Anquetin

\section{To cite this version:}

Virginie Anquetin. La domination mayorale - Analyser l'exercice du pouvoir des maires comme une société de cour. Revue française d'administration publique, 2015, 154 (2), pp.471. 10.3917/rfap.154.0471 . halshs-02269265

\section{HAL Id: halshs-02269265 \\ https://shs.hal.science/halshs-02269265}

Submitted on 22 Aug 2019

HAL is a multi-disciplinary open access archive for the deposit and dissemination of scientific research documents, whether they are published or not. The documents may come from teaching and research institutions in France or abroad, or from public or private research centers.
L'archive ouverte pluridisciplinaire HAL, est destinée au dépôt et à la diffusion de documents scientifiques de niveau recherche, publiés ou non, émanant des établissements d'enseignement et de recherche français ou étrangers, des laboratoires publics ou privés. 


\section{LA DOMINATION MAYORALE - ANALYSER L'EXERCICE DU POUVOIR DES MAIRES COMME UNE SOCIÉTÉ DE COUR}

Virginie Anquetin

Ecole nationale d'administration (ENA) | « Revue française d'administration publique »

2015/2 $\mathrm{N}^{\circ} 154$ | pages 471 à 488

ISSN 0152-7401

Article disponible en ligne à l'adresse :

http://www.cairn.info/revue-francaise-d-administration-publique-2015-2-page-471.htm

\section{!Pour citer cet article :}

Virginie Anquetin, « La domination mayorale - Analyser l'exercice du pouvoir des maires comme une société de cour », Revue française d'administration publique 2015/2 ( $\left.\mathrm{N}^{\circ} 154\right)$, p. 471-488.

DOI 10.3917/rfap.154.0471

Distribution électronique Cairn.info pour Ecole nationale d'administration (ENA).

(C) Ecole nationale d'administration (ENA). Tous droits réservés pour tous pays.

La reproduction ou représentation de cet article, notamment par photocopie, n'est autorisée que dans les limites des conditions générales d'utilisation du site ou, le cas échéant, des conditions générales de la licence souscrite par votre établissement. Toute autre reproduction ou représentation, en tout ou partie, sous quelque forme et de quelque manière que ce soit, est interdite sauf accord préalable et écrit de l'éditeur, en dehors des cas prévus par la législation en vigueur en France. Il est précisé que son stockage dans une base de données est également interdit. 


\title{
LA DOMINATION MAYORALE - ANALYSER L'EXERCICE DU POUVOIR DES MAIRES COMME UNE SOCIÉTÉ DE COUR
}

\author{
Virginie ANQUETIN \\ Docteure en science politique, Institut d'études politiques de Strasbourg, \\ Laboratoire Sociétés, acteurs, gouvernement en Europe (SAGE)
}

Résumé

Ce texte s'appuie sur l'analyse par Norbert Élias de la Cour royale pour comprendre les modes de fonctionnement et d'organisation structurels des municipalités quelles que soient leurs spécificités. Parce que la signature du maire est nécessaire pour déclencher tout acte municipal, l'institution mayorale est conçue pour lui donner les moyens de produire des stratégies organisationnelles de contrôle des élus dans sa majorité. En suscitant leur mise en concurrence et en interposant des experts personnellement attachés à son service entre les élus municipaux et l'appareil administratif, le maire évite le risque de la mobilisation de ses adjoints en dehors des élections.

\section{Mots-clefs}

Conseil municipal, maires, adjoints au maire, experts, services administratifs, société de cour

\begin{abstract}
- The power of the mayor over the members of the city council: municipalities as a court society - This article proposes to use Norbert Elias' concept of court society in order to understand the way municipalities work regardless of their specificities. The role of the mayor as it is conceived and practiced allows him to develop strategies of control of the other elected representatives of his political majority. By awakening competition between them and placing experts personally attached to him between these possible competitors and the administrative apparatus, the mayor reduces the risk of mobilization of the deputy mayors outside election periods.
\end{abstract}

\section{Keywords}

॥ City Council, mayors, deputy mayors, experts, local authority administrative services, court society

Comment un maire, personnage isolé, peut-il parvenir à s'imposer à la tête de sa municipalité et à s'y maintenir ? Pour expliquer l'autorité singulière du maire face à un conseil municipal de plusieurs dizaines d'élus et face à une administration comprenant parfois plusieurs milliers d'agents, diverses hypothèses ont été avancées. L'approche « présidentialiste » insiste sur son monopole en matière de production des «décisions » 
(Sorbets, 1983). L'analyse des «notables » urbains professionnels de la politique met l'accent sur la concentration et le cumul de fonctions publiques, politiques et partisanes dans les mains du maire (Garraud, 1989). Les travaux sur le leadership attribuent son succès à sa capacité de produire des collectifs pour le soutenir (Smith et Soberts, 2003). Mais quel mode de fonctionnement de l'institution permet d'expliquer l'ensemble de ces propriétés de l'exécutif municipal?

Analysant comment un homme, le roi de France, dont la capacité d'intervention directe était limitée, est pourtant parvenu à dominer une noblesse initialement armée, une bourgeoisie, des administrations, et un pays entier, Norbert Élias montre dans son ouvrage La société de cour que le fonctionnement de la cour royale constitue en lui-même un dispositif de contrôle fondé sur l'exploitation des concurrences et des jalousies. Le roi ne se contente pas uniquement de «diviser pour régner » : sa domination réside dans sa capacité à évaluer constamment les rapports de force entre ses rivaux et à équilibrer leurs puissances respectives de sorte à rendre toujours improbable leur mobilisation unifiée contre lui (Élias, 1985). Ainsi, les intérêts du roi sont portés et défendus par d'autres : plus son intervention est invisible, plus elle est efficace.

\section{Repères chronologiques}

1989 Élection de la liste socialiste menée par Catherine Trautmann à Strasbourg Premier adjoint : Roland Ries (ancien « chef» des rocardiens à Strasbourg) Majorité municipale : 44 élus dont 14 adjoints (Roland Ries, Jean Oehler, Norbert Engel, Marie-Hélène Gillig, Jean-Claude Petitdemange, JeanCharles Quintiliani, Claude Truchot, Robert Herrmann, Claude Kunz, Claude Lienhard, Robert Schumacher, Jean Klotz, Jean-Jacques Gsell, Michel Schmitt)

1995 Démission de René Hampé et de Fabienne Wassmer (mars-avril)

Réélection de Catherine Trautmann et de l'équipe socialiste (juin)

Premier adjoint : Roland Ries

Majorité municipale : 47 élus dont 19 adjoints (Roland Ries, Jean Oehler, Norbert Engel, Jean-Claude Petitdemange, Marie-Hélène Gillig, François Guichard, Jean-Charles Quintiliani, Claude Lienhard, Robert Herrmann, Jean-Pierre Fréani, Aziz Méliani, Constant Blum, Jean Klotz, Alain Kauff, Claudine Arnold, Jean-Jacques Gsell, Jean-Claude Richez, Philippe Forstmann, Michel Schmitt)

1996 Démission de Jean Oehler

1997 Nomination de C. Trautmann au ministère de la Culture du gouvernement Jospin

Élection de R. Ries maire - Premier adjoint : Marie-Hélène Gillig Majorité municipale : 47 élus dont 17 adjoints

1998 Démission de Philippe Forstmann

2000 Départ de C. Trautmann du gouvernement, puis réélection comme maire de Strasbourg par le conseil municipal

2001 Élection municipale, échec de la liste sortante 
Parce que les mairies sont, comme la société royale, des organisations polycéphales ${ }^{1}$ dont le chef est constamment menacé par des rivaux, il peut être pertinent de transposer la grille d'analyse de la société de cour pour éclairer les logiques sociales de fonctionnement et d'organisation de l'institution municipale. En effet, si en cours de mandat le maire n'apparait jamais pouvoir être renversé par des rivaux dans sa majorité, il est en revanche au moment de son renouvellement électif souvent contesté et parfois remplacé par l'un de ses adjoints, qui peut toujours présenter une liste contre celle du sortant, dans les villes moyennes ${ }^{2}$ comme dans les grandes villes ${ }^{3}$.

En nous appuyant en particulier sur l'étude des mandatures de Catherine Trautmann et Roland Ries à Strasbourg entre 1989 et $2001^{4}$, nous verrons comment l'analogie avec la société de cour nous permet de réinterpréter des éléments connus de la réalité municipale - la distinction entre adjoints et conseillers municipaux, la distribution de délégations, l'organisation d'un cabinet du maire, etc. - afin de saisir en quoi ces éléments contribuent tous de façon convergente à maintenir le maire en position d'arbitre des conflits dans la municipalité, de façon comparable au roi de France. Indépendamment des qualités propres de la personne qui l'incarne ou des spécificités des villes où elle se déploie, l'institution mayorale présente des aspects caractéristiques d'un gouvernement de cour. Le maire est un personnage central car toutes les nominations dépendent de lui, et pourtant il n'a pas besoin d'organiser personnellement toutes les interactions qui vont produire le contrôle réciproque de ses rivaux potentiels. Parce que l'institution confère à lui seul la capacité de promouvoir et de distinguer, il peut engendrer un appétit de récompense chez ses collaborateurs élus ou fonctionnaires qui, en retour, suscite un foisonnement d'activités à son service, pour contrôler ses adversaires et défendre son "intérêt particulier » électoral. La logique de la cour implique une faible dépense d'énergie pour le maire et une exposition minimale, même lorsqu'il choisit personnellement d'écarter

1. Norbert Élias suggère lui-même (Élias, 1985, 146-147) d'analyser comme de simples organisations des formes institutionnelles politiques ou économiques caractérisées par « un état de dépendance du personnage le plus libre et le plus puissant, lorsqu'il est installé à la tête d'une organisation géante », et de leur appliquer son analyse de la société de cour afin de comprendre le principe du maintien de l'autorité d'un seul sur tous.

2. Par exemple lors des élections municipales de 2014, s'affrontaient notamment des équipes menées par le maire sortant et par son ancien premier adjoint à Carpentras (Vaucluse), Creil (Oise), La Forêt-sur-Sèvre (Deux-Sèvres), Parçay-Meslay (Indre-et-Loire), Saint-Gobain (Aisne), Suresnes (Hauts-de-Seine), etc. L'ancien premier adjoint a battu le sortant à La Forêt-sur-Sèvre et Saint-Gobain, et l'a fait battre à Parçay-Meslay. Le maire sortant a été réélu à Creil, Carpentras et Suresnes.

3. Pierre Freyburger, ancien adjoint de Jean-Marie Bockel à Mulhouse, se présente contre lui en 2008 après son départ du Parti socialiste (PS). Ou encore, Hélène Mandroux et André Vézinhet, amis fidèles de Georges Frêche à la mairie de Montpellier, devenus professionnels de la politique grâce au maire dont ils étaient adjoints, placés par lui respectivement à la tête de la ville et du conseil général de l'Hérault, sont devenus par la suite ses ennemis politiques jurés. Pour un récit journalistique à charge contre G. Frêche voir : Maoudj, 2007. Pour un récit favorable au maire par un membre de son équipe, voir : Delacroix, 2007.

4. Ce texte s'appuie notamment sur un matériel recueilli dans le cadre d'une thèse de doctorat en science politique (entretiens, analyse des procès-verbaux des conseils municipal ou communautaire, d'articles de la presse régionale, d'archives partisanes, administratives et de cabinet) menée à partir de l'étude de l'entreprise politique de l'équipe socialiste qui conquiert puis dirige la mairie de Strasbourg de 1989 à 2001 (Anquetin, 2011). L'intérêt de mobiliser cette étude approfondie réside dans la possibilité de suivre en détail les trajectoires des élus et de reconstituer finement les rôles d'un ensemble variés d'auxiliaires dans la construction des jeux d'interdépendance, dont l'importance serait gommée si l'on s'en tenait aux discours ordinaires des acteurs politiques. L'intérêt tient aussi à la possibilité de restituer tant le cadre institutionnel stable du travail politique municipal que les effets des variations des rapports de force partisans sur les anticipations des acteurs et sur leurs stratégies - qu'elles soient couronnées de succès ou qu'elles échouent - dans un contexte local donné. Catherine Trautmann a été élue maire en 1989, réélue en 1995, puis a laissé son mandat à son premier adjoint Roland Ries en 1997 lorsqu'elle a été nommée dans le gouvernement Jospin. Elle est redevenue maire en 2000, puis l'équipe a perdu l'élection de 2001 au profit de la liste RPR-UDF concurrente. 
un concurrent ou de déclasser un collaborateur. Ce régime permet ainsi d'organiser un équilibre des tensions dans la municipalité qui, en dehors des élections municipales, met le maire entièrement à l'abri d'un « coup d'État » de ses adjoints. Que l'univers municipal soit fait de réglementations écrites et de statuts définis et protégés par la loi - loin d'être incompatible avec le caractère interpersonnel du fonctionnement curial - ne fait que mieux dissimuler derrière un paravent de neutralité bureaucratique les tensions humaines que ces règlements organisent (Élias, 1985, 147-148). Si l'attraction des électeurs requiert souvent la mise en scène du consensus entre gouvernants (Desage et Sibille, 2011) ou la publicité du consentement des « habitants » aux projets municipaux (Blatrix, 1999), l'institution municipale se présente comme une organisation hiérarchique reposant sur la capacité d'un chef à discipliner les effectifs.

Nous analyserons dans ce texte comment le maire contrôle la municipalité, en restituant les principales caractéristiques de la domination de type curial : la centralité d'un individu - ici, le maire -, sa capacité à discipliner ses rivaux en leur distribuant ou en leur refusant des « faveurs », et son aptitude à construire sa prééminence en produisant des antagonismes entre groupes différenciés. Nous montrerons tout d'abord que la situation du maire, premier personnage de la municipalité, procède de ses attributions institutionnelles qui lui confèrent une capacité unique à créer des positions dans la société municipale. Ensuite, nous examinerons successivement les dispositifs, emboîtés et évolutifs, par lesquels le maire domine la municipalité tout en minimisant son intervention directe. La présentation hiérarchique des positions dans la société municipale peut s'analyser comme un premier procédé permettant de discipliner les élus par la récompense. Tandis que, selon une logique proche de l'étiquette en vigueur à la cour royale, la majorité des élus est occupée à des tâches de figuration municipale - deuxième méthode de contrôle -, certains sont professionnalisés en politique sous condition de rester dépendants du maire (troisième dispositif). Toutefois l'accroissement inévitable de leurs ressources politiques tend à émanciper ces adjoints de la tutelle mayorale. Afin de limiter cette émancipation, le maire entretient des concurrences entre cadres partisans (quatrième dispositif), ou bien en suscite en faisant émerger un groupe rival à celui des adjoints, en particulier celui des collaborateurs de cabinet. La maîtrise de l'expertise municipale par des personnels sous la tutelle exclusive du maire (cinquième dispositif) favorise en effet son contrôle sur les actes et les prises des paroles des élus municipaux. La municipalité ressort à la suite de cet examen comme une structure concurrentielle comparable à la société de cour, assurant la domination hiérarchique du maire sur les élus et l'administration.

\section{LE MAIRE AU CENTRE DE L'INSTITUTION MUNICIPALE}

Parce que sa signature ${ }^{5}$ est nécessaire pour attribuer des titres et mandats aux élus municipaux, recruter dans l'administration, valider les délibérations et autoriser les dépenses, le maire est perçu comme central par tous ceux qui font partie de l'institution municipale : les cadres les plus haut placés et les agents d'exécution qui le reconnaissent

5. Inscrite dans le code général des collectivités territoriales, sa compétence est générale : le maire «prépare et exécute les délibérations du conseil municipal. Il conserve et administre le patrimoine communal, gère les revenus, prépare le budget, ordonnance les dépenses, dirige les travaux communaux, pourvoit aux mesures relatives à la voirie communale, procède aux adjudications, passe les marchés, représente la commune en justice, passe les actes de vente » (CGCT art. L.2122-21). 
comme «patron » de l'administration, les élus qui perçoivent l'attention préférentielle des journalistes et des électeurs en sa faveur, ou les collaborateurs de cabinet qui savent ne pouvoir s'imposer face à l'administration et aux adjoints sans son assentiment.

Ces prérogatives exceptionnelles du maire, qui font de lui un personnage capital et inamovible, ne résultent pas de spécificités culturelles localisées ou de dysfonctionnements organisationnels. Elles sont inscrites dans l'institution municipale même (Becquart-Leclercq, 1976) par laquelle le maire, autorité exécutive de la commune et « élu de la communauté » (George, 1989), est d'abord un agent de l'État, comptable de ses actes avant tout devant le gouvernement - qui seul peut le destituer ${ }^{6}$ en conseil des ministres - et non devant son conseil municipal. Malgré l'octroi d'un statut pour l'opposition en 1983, les lois de décentralisation n'ont fait que renforcer la personnalisation «monarchique » du pouvoir mayoral (Mabileau, 1995). La position du maire apparaît d'autant plus inexpugnable qu'elle semble bénéficier du soutien tacite des préfets et des juges des actes des collectivités - régulièrement dénoncé par certains membres de ces corps (Jouandet, 2013 ; Peraldi, 2014) -, et être le plus souvent acceptée par les électeurs, déférents à l'égard de sa «fonction politique » (Lascoumes, 2010). Les conseillers municipaux semblent impuissants à démettre leur maire quand bien même il serait complètement isolé : du maire socialiste imposé à la tête de sa ville en 1977, parvenu en quelques années à reprendre la main sur un conseil entièrement hostile (Dion, 1986, 69-75), au maire d'Aix-en-Provence élu en 1983, Jean-Pierre de Peretti Della Rocca, stupéfiant les élus municipaux lorsqu'il leur disait recevoir des instructions de la Vierge ${ }^{7}$.

A fortiori dans des circonstances ordinaires, l'asymétrie de position entre le maire et les élus municipaux (Le Bart, 2003, 149) est telle qu'il leur est plus profitable de travailler individuellement à accumuler des ressources politiques à l'intérieur du cadre prescrit par le maire plutôt que de l'affronter en dehors des élections. Quelles que soient les qualités morales ou les compétences stratégiques de la personne détenant le mandat mayoral, quelle que soit sa façon de s'approprier le rôle (Lagroye, 1997), le maire une fois élu peut librement user vis-à-vis de ses collaborateurs des droits à nommer, démettre et dépenser qui lui sont institutionnellement conférés (Gaxie et Lehingue, 1984) afin de les rendre dépendants de son entreprise politique. Il est en mesure de promouvoir et récompenser ses fidèles, d'entraver les ambitions de ses concurrents et de les punir, de favoriser ou d'empêcher des ascensions sociales et professionnelles.

En 1989, le maire de Strasbourg a ainsi pu s'attacher ses rivaux socialistes et l'ensemble des élus municipaux en leur distribuant, comme autant de titres de noblesse, non seulement des positions d'adjoint ${ }^{8}$ thématique, d'adjoint de quartier ou de vice-président de la CUS, ou des « secteurs » d'action publique, mais aussi des charges ouvrant droit à

6. Plus fréquente que sa rarissime révocation (d'une durée d'un an), la suspension du maire par le ministre de l'Intérieur pendant un mois sanctionne ceux qui s'opposent publiquement à une mesure gouvernementale. En 2000, cinq maires de petites communes sont suspendus pour refus d'organiser le référendum sur le quinquennat présidentiel. En 2004, Dominique de Villepin (UMP) ministre de l'Intérieur suspend Noël Mamère, député-maire de Bègles et candidat des Verts à l'élection présidentielle de 2002, pour le mariage politiquement clivant d'un couple homosexuel. Les maires réfractaires à la réforme des temps scolaires en 2014 ont été menacés de suspension par les ministres de l'Éducation Benoît Hamon et de l'Intérieur Manuel Valls (PS).

7. Voir Nathalie Funès, « Maryse Joissains est-elle à la hauteur ? », Le nouvel observateur, 10 janvier 2007.

8. Au $1^{\mathrm{e}}$ juillet 2010, les adjoints au maire d'une ville de 100000 à 199999 habitants reçoivent une indemnité brute maximale mensuelle de 2509 euros. Une majoration de $25 \%$ est applicable pour les chefs-lieux de département. Les indemnités de vice-présidents d'établissements intercommunaux de plus de 200000 habitants s'élèvent à 2756 euros mensuels. 
indemnité : les présidences ${ }^{9}$ de 18 sociétés d'économie mixte (SEM) et 7 établissements publics, les sièges de représentants de la ville dans 15 associations (dont le Racing-club de Strasbourg ou la SIG ${ }^{10}$ ), 12 organismes consultatifs (commission consultative économique de l'aéroport international de Strasbourg-Entzheim, etc.) ou établissements de formation supérieure (école nationale des ingénieurs des travaux ruraux, etc.). Le maire, seul, peut leur concéder une place au sommet de la hiérarchie municipale en leur accordant les revenus nécessaires pour se professionnaliser et les conditions pour apparaître comme des élus de premier plan face aux journalistes et aux électeurs.

« Mais qui l'a fait roi, Roland Ries ? Sans Catherine Trautmann, il n'existait pas ! » (Pierre Kling, direction de la communication à la ville de Strasbourg 1989-1995, cabinet du maire 1996-2001, entretien 18 mai 2004).

En outre, les rétributions symboliques, privilèges et honneurs liés aux positions élevées dans la majorité ou l'administration municipales (notoriété, signes de prestige tels que voiture avec chauffeur, places gratuites dans les manifestations culturelles, accès à une élite locale, sentiment d'être « aux commandes » d'une mairie, etc.), sont susceptibles d'engendrer des promotions sociales rapides qui inclinent les personnels politiques et administratifs à la docilité vis-à-vis du maire pourvoyeur de leur nouveau statut (Sawicki, 1998). Car tout aussi facilement qu'il peut promouvoir, le maire peut discrétionnairement retirer leurs délégations de signature et leurs mandats représentatifs aux élus jugés indisciplinés, ou même les révoquer ${ }^{11}$.

«On a eu un problème sur la première mandature avec une élue. Catherine Trautmann ne voulait pas une crise donc on ne l'a pas démissionnée, pour l'opinion publique elle a gardé son rang, mais elle n'avait plus rien à dire, on lui a enlevé son personnel et son pouvoir de signature » (Jean-Luc Marchal, cabinet du maire 1989-1990, entretien 26 mars 2003).

L'ensemble des postes supérieurs dans une municipalité est ainsi attribuable et révocable par le maire en place - pratique institutionnalisée dans la fonction publique territoriale par la règle de la «politisation fonctionnelle » (Roubieu, 1999b). Élus et responsables administratifs ne doivent leur rang ou leur emploi qu'à leur sélection par le maire, et sont amenés à quitter leur position en cas de remplacement du titulaire du mandat mayoral.

9. En 2015 à Strasbourg, présider la société gestionnaire de parking PARCus, le réseau Gaz de Strasbourg ou la Société d'aménagement et d'équipement de la région de Strasbourg rapporte environ 2470 euros mensuels, et la SEM Strasbourg-Événement, 1757 euros (Philippe Dossmann, « Échos du conseil : des SEM et des rémunérations », Dernières nouvelles d'Alsace, 22 avril 2015). Les rémunérations des élus municipaux sont légalement plafonnées à 8272 euros mensuels.

10. La ville de Strasbourg finance des équipes professionnelles de football (le Racing-Club de Strasbourg) et de basketball (Strasbourg Illkirch Graffenstaden Basket).

11. Selon le CGCT, le maire peut à tout moment mettre fin aux délégations qu'il a consenties « sous réserve que sa décision ne soit pas inspirée par des motifs étrangers à la bonne marche de l'administration communale ». Mais selon un arrêt du conseil d'État « la rupture de solidarité avec la politique du maire » suffit à motiver le retrait de la délégation de signature. Si les maires de Strasbourg entre 1989 et 2001 n'ont jamais retiré la délégation de premier adjoint, la pratique n'est pas marginale. Les premiers adjoints des listes élues ou réélues en 2014 sont déjà révoqués à Argelès-sur-Mer (PS-PRG) dans les Pyrénées-Orientales, à Meythet (divers gauche) en Haute-Savoie, à Avesnelles (sans étiquette) dans le Nord, à Hayange (FN) en Moselle, etc., auxquels il faut ajouter ceux qui ont préféré démissionner (à Merville en Haute-Garonne, à Baden dans le Morbihan, La Châtre dans l'Indre, etc.). 
Cette capacité de nomination dépasse les frontières de la municipalité. Le contrôle de l'institution municipale fournit également au maire des ressources pour contenir l'expression de contestations qui pourraient émaner de son parti. Parce qu'il peut augmenter le nombre des adhérents, le maire d'une capitale régionale est en mesure de contrôler les votes ou les investitures (Juhem, 2006) et de choisir un dirigeant partisan chargé de veiller à l'assèchement des critiques internes. Pris dans les jeux municipaux (Lefebvre, 2004, 2007), le parti est dirigé en coulisse par le maire.

La puissance du maire est telle que les élus comme les agents administratifs anticipent que l'amélioration de leur position ne peut s'obtenir que par l'acceptation de la chaîne hiérarchique. Le maire peut ainsi, de façon routinisée, déployer d'autres moyens que la sanction publique pour discipliner élus et personnels administratifs dans la municipalité.

\section{LA PRÉSENTATION HIÉRARCHIQUE DE « L'ÉQUIPE MUNICIPALE » : UN OUTIL DISCIPLINAIRE}

Dans la municipalité, une subtile gradation des prestiges et responsabilités va de la position de conseiller municipal à celle d'adjoint aux finances ou de premier adjoint. C'est dans cette hiérarchie apparente, qui permet au maire de concéder à chacun un statut valorisant ajusté à ses prétentions en même temps que de dissimuler les inégalités réelles de position, que réside l'efficacité disciplinaire de cette organisation. La hiérarchie municipale n'est pas un cadre fixé par la loi ou un dispositif de rationalisation administrative. Elle est la résultante de ce travail mayoral de régulation, par la récompense ou la punition, des fidélités et des antagonismes des élus majoritaires.

Par l'attribution des titres d'adjoints et de conseillers municipaux avec ou sans délégation, le maire peut opérer des affichages spécifiques selon une logique de casting : il confie à des colistiers aux propriétés professionnelles ou sociales particulières des délégations supposées bénéficier de leur expertise, et affiche ainsi des thématiques découlant des rapports de force partisans. Mais les positions d'adjoint ne sont pas distribuées dans le souci premier d'ajuster les compétences des élus à un secteur administratif. Elles le sont en fonction de l'efficacité disciplinaire anticipée de ces rétributions. Les cadres partisans nommés adjoints se voient attribuer des « secteurs » dont le découpage - qui n'a jamais fait l'objet d'une standardisation nationale ${ }^{12}$ - doit contribuer à leur fidélisation et entretenir leur dépendance personnelle vis-à-vis du maire.

À Strasbourg après l'alternance de 1989, la distribution des délégations par le nouveau maire qui revendique une identité " socialiste moderne ${ }^{13}$ émerge de ces deux ensembles de contraintes. Les intitulés des délégations reflètent l'adaptation de la municipalité aux nécessités de la compétition partisane, en contribuant à l'affichage des préoccupations « gestionnaires », « de droite » («finances », « urbanisme » ou « affaires économiques »), « de gauche » (« logement social ») ou « environnementales » de la nouvelle équipe, dans un contexte où les Verts obtiennent près de $13 \%$

12. La généralité des labels (« environnement », « européanisation », etc.) homogénéise à l'excès des usages pourtant localement très différenciés (Bué, Desage, Matejko, 2004, 77-78).

13. Se revendiquant du courant Rocard pendant les années 1980, C. Trautmann a incarné avec succès un positionnement « recentré » du PS à Strasbourg en promouvant une étiquette « socialiste moderne ». 
des suffrages. Mais les délégations découlent aussi de la capacité des cadres socialistes à prouver au maire leur fidélité et à lui montrer comment ils peuvent contribuer à son entreprise politique.

«En 1989, j'étais en $13^{\mathrm{e}}$ position sur la liste [...] Or au départ j’étais beaucoup plus loin [...] Donc je suis allé voir Trautmann. Je lui ai dit "écoute, ça ne va pas". Je connaissais quand même beaucoup de militants dans la fédération et à peu près toute la presse régionale, c'était pour la plupart des copains [...] Donc j'avais un certain nombre de circonstances qui faisaient que ce n'était pas aberrant que je sois à une meilleure place [...] C'est là qu'elle m'a dit : “bon, tu seras à la $13^{\mathrm{e}}$ place”. J'y ai été mis aussi parce qu'il y avait l'idée que je serais un type loyal et que je bosserais [...] Je me suis retrouvé au sport, mais je le dis toujours : quinze jours avant les élections, je jetais, sans le lire, le cahier des sports des Dernières nouvelles d'Alsace » (Robert Herrmann, adjoint aux sports 1989-2001, conseiller général du canton 1 de Strasbourg depuis 2001, premier adjoint 2008-2014, président de Strasbourg Eurométropole depuis 2014, entretien 6 novembre 2004).

Le maire est toutefois en mesure de confier une délégation à un adjoint en vue mais en lui donnant l'appui d'un service qui n'a pas la possibilité de distribuer des subventions ou autorisations, ou bien d'un service financé et doté de personnel, mais dont les budgets ne sont pas affectables discrétionnairement, ou même, de ne pas lui donner d'autorité sur un service administratif, limitant de fait l'accroissement autonome des ressources politiques de l'élu (Borraz, 1998, 127). L'organigramme officiel ne reflète ainsi jamais entièrement la hiérarchie municipale réelle ${ }^{14}$ qui fait au contraire l'objet de luttes constantes - d'autant plus que des « personnalités de la société civile » ou des membres du cabinet plus « proches » du maire peuvent remplacer les adjoints en titre dès l'élection suivante.

\section{INSTAURER UNE CONTRAINTE VALORISANTE DE FIGURATION MUNICIPALE}

Les adjoints et conseillers municipaux sont donc incités à une soumission conciliante. L'adoption de cette posture est facilitée en particulier par leur occupation à des tâches régulières et obligatoires de figuration municipale, comparables au fonctionnement de l'étiquette à la cour du roi de France décrite par Norbert Élias (Élias, 1985, 82) : il leur est difficile de s'en affranchir sous peine de se trouver hors-jeu et de ne jamais avoir l'opportunité d'améliorer leur position. Être choisi pour représenter ou même simplement accompagner le maire lors d'inaugurations ou de visites variées, ou pour animer des réunions publiques, constitue un outil de valorisation permettant d'apparaître devant les journalistes en incarnant le rôle de l'élu «bâtisseur» ou « entrepreneur » (Le Bart, 1992) au « service des habitants » (Anquetin et Freyermuth, 2008). La mise à disposition

14. À Paris sous les mandatures de Jacques Chirac, la préséance nominale des adjoints sur les conseillers municipaux est souvent démentie dans les faits (Haegel, 1994). Pour un récit journalistique, voir : Madelin, 1997. 
de « collaborateurs de groupe », officiellement chargés d'assister les élus municipaux, peut être interprétée comme l'un des dispositifs prescripteurs de cette posture de représentation du maire auprès des électeurs, qui doit amener les élus à figurer honorablement dans la municipalité sans transgresser les limites du rôle imparti. Dans la même logique, les élus peuvent se voir aussi attribuer des tâches de « gestion » sous la forme de participation à des réunions régulières (bureau hebdomadaire, comité de suivi, comité de pilotage, etc.), quand bien même rien de décisif n'y serait décidé. Ces réunions permettent, le cas échéant, au maire ou à ses auxiliaires d'orchestrer l'enlisement d'une réalisation et, à travers elle, la marginalisation de l'élu qui la porte.

Comme à la cour royale, la vie municipale est faite de la comparaison perpétuelle entre soi et les autres membres de son groupe, cadres administratifs ou élus, favorisée par leur fréquentation quotidienne. La cohabitation de l'ensemble des personnels municipaux en un centre unique les contraint à l'estimation constante de leurs situations et privilèges respectifs - ce qui, comme les courtisans à Versailles, les engage davantage dans une lutte pour l'amélioration de leur statut, ou, en empruntant au vocabulaire de Norbert Élias, de leur prestige dans la municipalité. Recevoir chaque matin un exemplaire du journal local sur sa table de travail, bénéficier d'une surface de bureau plus vaste que ses concurrents, travailler près de l'étage du maire ou au contraire dans un bâtiment éloigné (Roubieu, 1999a, 252), être invité à des séances de travail ou à des moments de convivialité, sont autant d'indicateurs de l'évolution du rang relatif de celui qui bénéficie de ces marqueurs de prestige dans la municipalité. Loin de ne constituer que des satisfactions narcissiques, ces indicateurs signalent à tous, personnels administratifs ou élus, la faveur ou la défaveur des uns et des autres, voire leur « mise au placard », et ils canalisent ainsi les comportements de coopération ou au contraire de prise de distance qui contribuent à faire exister ou disparaître l'activité de toute personne dans l'administration municipale.

\section{ACCORDER UNE PROFESSIONNALISATION CONDITIONNELLE}

Pour dépasser le rôle de «figurant » et prétendre infléchir l'usage des ressources municipales, et donc pour exister politiquement, consacrer la totalité de son temps professionnel à son mandat constitue une nécessité (Marrel et Payre, 2001). La hiérarchie réelle des positions dans la municipalité n'est pas donnée par l'organigramme, mais par le degré de professionnalisation politique ${ }^{15}$ de chaque membre de la majorité.

La professionnalisation d'un élu municipal requiert le cumul de plusieurs revenus : non seulement ceux procurés par un mandat municipal (y compris intercommunal), mais aussi par la représentation de la mairie dans un organisme paramunicipal ou la conquête d'un autre mandat local. Par conséquent le maire est contraint de travailler à contrôler toutes les ressources de professionnalisation élective disponibles, notamment les investitures partisanes aux élections cantonales, régionales, législatives ou sénatoriales, ce qu'il

15. Devenir professionnel de la politique implique une aspiration au schéma idéal suivant : tirer sa subsistance de l'activité politique pour s'y consacrer à temps plein, ce afin d'accroître de façon autonome ses ressources, savoir-faire et savoir-être politiques, de façon à limiter les ruptures dans la carrière occasionnées par l'aléas électoral. Sur les enjeux et les luttes de classification de l'activité politique pérenne, voir notamment : Lehingue, 1999. 
obtient par la production systématique des effectifs militants de son parti (Hû, 2014). Il doit non seulement se procurer toujours de nouvelles ressources de professionnalisation afin de les répartir, mais aussi occuper lui-même une position toujours mieux rétribuée que celle de ses rivaux et alliés dans la municipalité - sous peine d'encourager les défections dans son camp. La gestion curiale des équipes municipales en voie de professionnalisation tend ainsi à stimuler la conquête de nouveaux mandats et leur cumul (Marrel, 2003 ; Paoletti, 2007), « vertical » ou « horizontal ».

Toutefois, loin d'accorder les conditions d'une professionnalisation politique de façon systématique ou inconditionnelle, le maire est au contraire amené à jouer continuellement sur la promesse de son obtention tout en contenant autant que possible sa réalisation. Le maire agit en permanence sur ses rivaux par un jeu de faveurs et de défaveurs organisant leur élévation et leur stagnation dans la carrière afin d'éviter la coalition de leurs forces et de ralentir la survenue de dissidences pourtant inévitables à long terme. Le groupe des adjoints rassemble donc des élus entièrement sous contrôle du maire et quelques professionnels susceptibles de devenir ses rivaux. Au cours des mandatures de C. Trautmann et R. Ries à Strasbourg entre 1989 et 2001, 6 cadres socialistes ${ }^{16}$ et 2 «personnalités de la société civile ${ }^{17}$, connaissances personnelles du maire lui étant entièrement redevables de leur nouvelle situation, ont été professionnalisés. En 1989, la municipalité comptait pourtant 14 adjoints sur 44 élus majoritaires en plus du maire (dans un conseil municipal d'une soixantaine d'élus), 19 sur 47 en 1995, 17 en $1997^{18}$. Ces professionnalisations ont constitué le support des affrontements internes à la municipalité strasbourgeoise (C. Trautmann contre son premier adjoint R. Ries et contre M.-H. Gillig première adjointe de R. Ries). Une fraction des adjoints professionnalisés (J.-C. Petitdemange, J.-P. Fréani et J.-C. Quintiliani) a fait scission et a présenté sa propre liste à l'élection de 2001, qui s'est soldée par l'échec des sortants.

Pour limiter les ressources politiques des adjoints les plus professionnalisés, le maire peut mener une stratégie consistant après sa réélection à justifier par les nécessités de la conjoncture électorale le non renouvellement de leur délégation (Borraz, 1998, 120 ; Troupel, 2006) ${ }^{19}$ et les remplacer en suscitant des candidatures en dehors des réseaux partisans. Les « personnalités de la société civile », dont le maire peut encourager la professionnalisation politique, ont pour propriété de n'avoir aucune expérience militante dans le parti et de dépendre entièrement du maire pour le maintien de leur investissement en politique - et donc d'être dépourvues de ressources politiques à leur arrivée dans la majorité. Les délégations attribuées peuvent alors être reformulées et les liens personnels qui ont pu s'établir entre élus professionnels et cadres administratifs au cours du mandat précédent, affaiblis ou supprimés.

16. Roland Ries premier adjoint aux transports puis maire, Jean Oehler deuxième adjoint au logement et développement social urbain, Robert Herrmann adjoint au sport, Jean-Charles Quintiliani adjoint à la sécurité, Alain Kauff adjoint au logement social, Jean-Pierre Fréani adjoint à la circulation et au stationnement. L'adjoint aux finances Jean-Claude Petitdemange, préfet hors cadre, était financièrement indépendant du maire.

17. L'adjoint à la culture Norbert Engel, enseignant en philosophie devenu inspecteur général des Affaires culturelles en 1998, et l'adjointe à l'action sociale et au personnel Marie-Hélène Gillig, responsable d'associations dans le secteur social devenue députée européenne socialiste de 1999 à 2004.

18. En 1997, la municipalité a perdu deux adjoints : R. Ries, élu maire à la place de C. Trautmann devenue ministre de la Culture, et J. Oehler, mis en cause judiciairement et ultérieurement acquitté de toute charge.

19. A Besançon, O. Borraz note que « [en 1989], seuls 3 adjoints ont été élus pour la première fois en 1977, outre le maire [Robert Schwint] ». Toutes les configurations politiques ne favorisent toutefois pas la mise en œuvre de cette stratégie. 
À Strasbourg en 1995, C. Trautmann n'a pas saisi l'opportunité de sa réélection au premier tour ${ }^{20}$ pour écarter certains de ses adjoints bénéficiant alors d'une notoriété personnelle, et pour professionnaliser de nouveaux élus issus de la «société civile » qui lui auraient été entièrement redevables de leur statut électif ${ }^{21}$. " L'exécutif » compte alors 8 nouveaux adjoints ${ }^{22}$ sur 19 , mais aucun n'occupe cette fonction à temps complet. Seuls 4 des adjoints au maire n'étaient pas déjà élus durant la mandature précédente $^{23}$. Sur les 14 adjoints nommés en 1989, 11 sont finalement reconduits dans leur délégation.

C'est alors le poids politique des adjoints professionnalisés qui tend à dicter l'intitulé des délégations, à organiser l'intervention municipale en segments administratifs placés sous leur supervision, et à figer les modalités administratives de gestion des problèmes politiques auxquels la municipalité est confrontée - mobilisations oppositionnelles, traitement journalistique défavorable, etc., affaiblissant la capacité de C. Trautmann à maintenir efficacement des stratégies de limitation des ressources politiques de ses adjoints désormais plus indépendants.

Afin d'abaisser leurs gains de notoriété et d'influence, le maire et ses auxiliaires développent alors une autre stratégie qui consiste à inhiber les dépenses municipales et «l'action publique ». Toutefois, à moins que l'implantation du maire soit stable et ancienne - que son ascendant sur son territoire électif soit fort -, cette stratégie compromet sa capacité à se montrer perpétuellement « actif et compétent » et à engendrer des soutiens électoraux.

\section{ORGANISER LES CONCURRENCES ENTRE ÉLUS}

Pour contrôler les élus municipaux, en particulier ceux qui, élus à un mandat cantonal, peuvent développer une notoriété et des soutiens électoraux propres, le maire peut appuyer ou construire des concurrences internes à la municipalité, tout comme le roi à la

20. Les anticipations politiques des membres de la municipalité sont alors marquées par une croyance émergeant des rapports de force électoraux à cette époque : Strasbourg, «ville de droite », serait toujours hostile à des entreprises politiques « socialistes ». L'équipe autour de C. Trautmann tend donc à considérer qu'il est prioritaire de ne pas donner prise au principal thème critique utilisé par l'opposition RPR et UDF, « l'incompétence gestionnaire » de la municipalité PS, et de donner au contraire des gages de « compétence » et de «bonne gestion ». Après la réélection de l'équipe sortante en 1995, les adjoints professionnalisés au premier mandat ont utilisé - avec succès - cet argument de leur « compétence » acquise au cours du mandat échu, qu'ils présentent comme un élément du crédit gestionnaire de la municipalité socialiste, pour faire pression sur le maire afin d'obtenir le renouvellement de leur délégation. Or, au lendemain de la réélection d'un responsable d'exécutif sortant au premier tour, la capacité de ses adversaires à le critiquer est très affaiblie (pour une analyse générale des conditions de construction des controverses dans l'espace public, voir : Juhem, 2014). On peut donc penser que la configuration politique en 1995 aurait au contraire facilité l'adoption d'une stratégie de marginalisation de ces adjoints par le maire.

21. Trois élus en particulier rentraient dans cette catégorie : Jean-Claude Richez, conseiller municipal de 1989 à 1995, enseignant en histoire et ancien militant à la LCR, François Guichard, magistrat, et Philippe Forstmann, enseignant en IUT et ancien Directeur régional des affaires culturelles. Aucun de ces nouveaux adjoints, recrutés par l'entourage de C. Trautmann, ne le sera à plein temps à la fin du mandat.

22. François Guichard, Claudine Arnold, Philippe Forstmann, Jean-Claude Richez, Jean-Pierre Fréani, Aziz Méliani, Constant Blum, Alain Kauff.

23. Alain Kauff, adjoint au logement social, Nicole Dreyer, adjointe à l'éducation et François Guichard, adjoint prévention et médiation, Philippe Forstmann, adjoint à l'enseignement et aux cultes, qui démissionne en 1997. 
cour (Haroche, 1991), lesquelles ont pour effet de canaliser l'énergie sociale et politique de ses rivaux dans des luttes mutuelles. Ces luttes résultent d'abord des inégalités de position organisées par le maire, qui utilise le découpage des délégations, l'attribution de l'autorité sur des services administratifs et donc sur des budgets, ou la répartition des investitures disponibles, de façon à placer les élus en situation de concurrence objective les uns avec les autres - ce qui a pour effet de contenir l'accroissement des ressources politiques des plus ambitieux.

Malgré une forte présence des adjoints dans la mise en scène de l'action sectorielle de leurs municipalités, C. Trautmann et R. Ries ne font pas exception. L'analyse de la place de l'adjoint Jean Oehler puis de son fils Serge dans l'équipe municipale montre que, si ceux-ci ont été encouragés à entretenir leur implantation électorale construite depuis les années 1970 dans les quartiers populaires de Hautepierre et Cronenbourg, l'entourage du maire a eu le souci permanent de ne pas permettre à l'adjoint puis à son fils d'étendre un succès politique localisé ou d'en monopoliser le bénéfice électoral. En 1989, J. Oehler ${ }^{24}$, tête de liste socialiste à l'élection municipale précédente et allié à C. Trautmann pendant la campagne, ancien conseiller général élu à Hautepierre et Cronenbourg, est nommé deuxième adjoint délégué au « logement, développement social urbain et opérations programmées d'amélioration de l'habitat » afin de mettre en œuvre la rénovation de l'habitat social dans les quartiers populaires - mesure permettant de capter le vote des électeurs qui y résident. Mais la compétence en matière de transactions immobilières et d'urbanisme appartient toujours strictement au maire. En outre, invoquant la nécessité d'une intervention transversale de « mission », le maire n'accorde aucun budget propre à la petite équipe nouvellement constituée du « développement social urbain »(DSU), ce qui fait de l'activité de sa directrice une succession de luttes contre les autres services - jusqu'à la suppression du DSU en 1995. Après la réélection de l'équipe, Serge Oehler remplace son père Jean mais il n'est accepté que comme simple conseiller municipal. Candidat dans le même canton dès 1992, élu en 1998, il ne se voit jamais octroyer une délégation d'adjoint thématique ou de quartier, confiée à un autre.

«Ceux qui sont élus conseillers généraux deviennent des personnages complètement incontournables dans la vie politique locale, donc il faut avoir certaines vigilances, repérer là où on a un îlot de pouvoir naissant, et voir ce qu'on peut faire. C'est pour ça que Serge Oehler n'a jamais été adjoint au maire : là, c'était bien joué. On a pu jouer l'équilibre [...] en mettant élu de quartier Constant Blum, qui n'était pas le conseiller général. L'élu de quartier a beaucoup souffert, il a eu des différends à n'en plus finir avec la famille Oehler, mais il a effectivement, sans doute à son corps défendant, évité que Serge Oehler ne prenne trop [de place] » (Pierre Kling, direction de la communication 1989-1995, cabinet 1997-2001, 30 juin 2004).

En délimitant les attributions de chaque élu avec l'objectif qu'aucun adjoint n'ait de façon prolongée le contrôle sectoriel de services administratifs, ou la capacité d'apparaitre devant les journalistes et les électeurs comme l'auteur d'une «politique » spécifique, ou encore la possibilité d'établir des loyautés électorales par la distribution indépendante de subventions, autorisations et services, le maire limite l'accroissement de leurs ressources politiques pendant la durée du mandat et leur capacité à s'autonomiser de son entreprise politique. 


\section{ORGANISER LA DÉPENDANCE DES ÉLUS VIS-À-VIS DES « EXPERTS »}

Pour empêcher l'autonomisation politique des élus, le maire peut leur opposer des groupes de collaborateurs « experts »- analogues aux ministres, non issus de la haute noblesse, dont le roi s'entourait (Élias, 1985, 120) - placés au cabinet ou dans les services administratifs. En recrutant dans l'administration ou au cabinet des « spécialistes », conseillers en communication ou attachés de presse (Legavre, 1989 et 1992 ; Georgakakis, 1995) chargés officiellement de faciliter les relations avec les journalistes et d'améliorer leur couverture des réalisations municipales, et des conseillers juridiques et financiers supposés « sécuriser les décisions » (Courty, 2005), le maire organise la dépendance des élus envers des professionnels choisis par lui. La discipline que ces dispositifs produisent est en effet légitimée auprès des élus par le crédit « expert » (budgétaire, juridique, technique) ou «managérial » (Rowell, 2006) de ces auxiliaires mayoraux. Mais ce qui fait leur employabilité à ces postes est tout autant leur recrutement en dehors des réseaux politiques locaux et la fragilité de leur statut (emplois « fonctionnels » ou contrats de cabinet), qui les rendent entièrement dépendants du maire pour leur promotion professionnelle et politique, et les font échapper à l'emprise des adjoints.

Cette stratégie organisationnelle permet au maire de n'apparaître que comme l'arbitre et non l'initiateur des conflits récurrents entre élus et collaborateurs « experts ». Les conseillers de cabinet en particulier, par leur situation surplombante due à leur proximité avec le maire, sont en mesure de redéfinir la division du travail politique (Dulong, 1997) et de limiter l'accès des élus aux ressources administratives et expertes de l'institution. Ainsi, ces collaborateurs freinent l'établissement de relations sectorielles stables et personnalisées entre adjoints, cadres administratifs et experts, et donc limitent leur capacité à produire des fidélités électorales à partir de « politiques municipales » menées en leur nom propre.

À Strasbourg, dans la configuration d'alternance en 1989, le maire a pu imposer à des adjoints sans alliés administratifs et sans expérience antérieure de gestion municipale de s'en remettre à ses collaborateurs personnels. Bénéficiant d'un crédit de neutralité « technique » et d'expertise étatique (anciens élèves de l'ENA, magistrats, auxiliaires des ingénieurs des Ponts et Chaussées auprès de la DATAR, ancien inspecteur du Trésor, etc.) qui leur permet de superviser l'activité de l'administration et de dominer les élus municipaux, réputés plutôt «à droite », les collaborateurs de cabinet ont favorisé l'établissement du positionnement « socialiste moderne » « gestionnaire » de Catherine Trautmann et la réalisation d'un tramway contre les options défendues par certains des principaux cadres partisans dans la majorité (tramway «Alsthom » ou même simple amélioration du réseau de bus).

« La constitution d'un cabinet fort - c'est une chose à ne pas répandre dans Strasbourg ! - était structurellement une défiance à l'égard des adjoints » (Jean-Michel Guibert, conseiller technique « budgétaire » au cabinet de C. Trautmann à Strasbourg 1989-1992, entretien 28 mars 2003).

L'importance du dispositif de contrôle « expert » se mesure aussi au nombre de personnes qu'il mobilise. À Strasbourg, bien que le maire ne soit autorisé à embaucher que 9 conseillers de cabinet ${ }^{25}$, les entretiens avec les collaborateurs mayoraux font ressortir qu'entre 1989 et 1995, le cabinet a indirectement compté jusqu'à une cinquantaine de personnes, sans qu'aucune embauche n'ait été faite de façon dérogatoire ou illégale.

25. 7 au titre de la mairie, et 2 au titre de la communauté urbaine. À Strasbourg la fusion des administrations municipale et communautaire dès la création de la CUS en 1966 fait du maire l'élu dominant sur ces deux institutions. 
Le nombre de ces auxiliaires mayoraux n'est en effet jamais véritablement précisé ou restreint par les réglementations : si l'effectif des cabinets est plafonné et ne permet pas de placer derrière chaque cadre administratif ou chaque adjoint un professionnel agréé par le maire, l'absence de limitation de la taille des administrations, la variété des postes qu'ils peuvent y occuper et le recrutement d'auxiliaires qui intègrent ce travail collectif au titre d'autres mandats détenus par le maire (Beauvallet, 2014) facilitent l'extension d'un contrôle mayoral délégué. La position institutionnelle du maire lui confère donc une capacité à augmenter son autorité au moyen de personnels légitimes dont les rhétoriques professionnelles fonctionnent comme des outils de subordination de la municipalité.

Cependant, le cabinet, dispositif centralisé et « technique » de captation de «l'expertise », tend à perdre de sa force à mesure de la reprise en main de l'administration, du renouvellement de ses principaux cadres administratifs, et des « arbitrages » que le maire rend en faveur des adjoints - et donc contre ses collaborateurs.

Constatant à Strasbourg l'indépendance croissante des adjoints vis-à-vis du cabinet, C. Trautmann et ses auxiliaires s'appuient sur le succès de sa réélection au premier tour en 1995 pour relocaliser la maîtrise des ressources municipales d'expertise dans l'administration en y transférant ses collaborateurs techniques ${ }^{26}$ et en recrutant de nouveaux cadres susceptibles d'effectuer le même travail de contrôle des élus depuis l'administration. Pour encadrer les adjoints qui ont acquis des ressources politico-administratives au cours du premier mandat, le nouveau directeur général des services édifie une « direction des affaires juridiques » chargée non seulement du contentieux de l'administration, tâche ordinaire de ce service, mais aussi de l'évaluation juridique ou budgétaire des demandes d'allocation des ressources municipales. Construit en particulier contre une « direction des finances » dont le responsable est jugé plus fidèle à l'adjoint J.-C. Petitdemange qu'au maire, ce nouveau service interpose à nouveau des auxiliaires mayoraux entre les adjoints et les services, dans les formes acceptables de la « sécurité des décisions » et de la promotion de méthodes managériales, souvent indissociablement « juridiques » ou « budgétaires » et «politiques ». L'efficacité de cette stratégie de dissolution des alliances entre segments de l'administration, « experts » et élus, a été toutefois amoindrie par la position dominée du secrétaire général par rapport au directeur de cabinet héritée de la configuration du premier mandat, par le nombre réduit de collaborateurs non liés au service des adjoints que le nouveau directeur général s'autorise à recruter, et surtout par la reconduction à l'identique des délégations des adjoints. L'affrontement entre les partisans des deux maires R. Ries et C. Trautmann lorsque celle-ci quitte le gouvernement Jospin en $2000^{27}$ rend visible la division des ressources d'expertise de

26. En particulier, la conseillère technique spécialisée dans les implantations d'entreprises et les affaires immobilières est placée à la tête d'une nouvelle direction du développement de l'aménagement. Le directeur de cabinet, prématurément décédé pendant la campagne de 1995, devait occuper le poste directeur général des services. Le conseiller «budgétaire » a quitté le cabinet en 1993.

27. C'est C. Trautmann elle-même, maire en fonction, qui, suite à sa nomination comme ministre en 1997, a confié son siège à son premier adjoint et n'a conservé qu'une position de conseiller municipal - étant d'ailleurs l'un des deux seuls ministres à respecter cette consigne gouvernementale (voir Guillaume Perrault, «Le non-cumul : les aléas d'une règle », Le Figaro, 31 août 2009). Son départ a entraîné des défections dans son propre camp et a permis au nouveau maire d'accroître concurremment les fidélités à sa personne et son entreprise politique. Toutefois, en partie pour ne pas être publiquement accusé de « traîtrise », R. Ries alors maire a conservé une part importante des collaborateurs de son prédécesseur. L'entourage mayoral (le cabinet et, dans une moindre mesure, la direction de l'administration) est donc apparu constitué de deux camps opposés, l'un en faveur du maire en place, l'autre remobilisé par C. Trautmann, qui finit par l'emporter dans les votes internes à la fédération socialiste. 
la collectivité - principalement entre la direction « juridique » du directeur général des services et la direction « financière » de l'adjoint aux finances - et précipite la division publique de l'équipe.

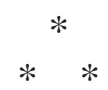

L'analogie avec le fonctionnement de la cour royale permet de percevoir que la domination du maire sur la municipalité et l'administration au cours de son mandat résulte d'un travail collectif qui repose sur sa capacité à mettre en concurrence des groupes ou des individus grâce aux ressources que l'institution lui confère spécifiquement. Le maire est lui-même contraint par cette organisation car il doit toujours apparaître comme étant le seul à pouvoir améliorer ou défaire la position de chacun, en particulier celle de ses rivaux, afin de ne pas encourager la formation d'une coalition adverse dans son propre camp. Toutefois, loin d'être une propriété du maire en tant qu'individu, son autorité est produite par un ensemble d'activités sociales, indissociablement administratives, techniques et politiques, que sa position lui permet de susciter et dont elle lui procure le bénéfice, mais qu'il ne contrôle et n'initie pas nécessairement unes à unes. La hiérarchie municipale officielle participe de ce contrôle collectif parce que sa plasticité permet de dissimuler l'évolution des inégalités réelles des ressources politiques de chacun derrière la satisfaction d'un statut public valorisant et stable. À ce statut est attaché un ensemble de tâches prescrites de représentation du maire ou de gestion administrative, dont l'exécution conforme favorise l'obtention de récompenses accordées au cas par cas. Cette organisation tend à faire fonctionner tous les protagonistes municipaux comme autant d'instruments de surveillance et de contrôle des uns par les autres au bénéfice du maire. Même lorsqu'elle est accordée, la récompense ultime que constitue la professionnalisation politique d'un élu n'affranchit pas celui-ci de la domination mayorale : la maîtrise que l'institution procure au maire sur l'ensemble des ressources de professionnalisation élective permet à celui-ci de susciter un concurrent qui s'opposera nécessairement à l'adjoint jugé trop ambitieux parce que sa survie politique dépendra de sa capacité à le contenir. Enfin, parce que «l'expertise » est une ressource politique, le maire peut entraver la progression de ses rivaux dans la municipalité en les rendant dépendants de «spécialistes » qui lui sont personnellement attachés. Ainsi, l'institution municipale, qui permet l'organisation du contrôle d'un seul sur tous, engendre également les « dysfonctionnements » administratifs résultant de la mise en concurrence des élus municipaux entre eux. L'analyse curiale de l'institution municipale complète les travaux sur le pouvoir des maires en permettant de comprendre comment les multiples interactions, obligations et interdépendances qui s'établissent de façon spécifique à chaque municipalité et à chaque territoire sont pourtant le produit d'un même fonctionnement institutionnel.

\section{Références bibliographiques}

Anquetin, Virginie (2011), La construction électorale des politiques municipales. Travail politique de conquête et de gestion d'une capitale régionale (Strasbourg 1973-2001), Thèse pour le doctorat en science politique, sous la direction de Renaud Dorandeu, Université de Strasbourg. 
Anquetin, Virginie, et Freyermuth, Audrey, dir. (2008), La figure de l' « habitant». Sociologie politique de la «demande sociale », Rennes, Presses universitaires de Rennes.

Beauvallet, Willy (2014), « Pour une sociologie des auxiliaires politiques et parlementaires. L'exemple de l'Assemblée nationale », in Mazeaud, Alice, dir. (2014), Pratiques de la représentation politique, Rennes, Presses universitaires de Rennes, p. 163-178.

Becquart-Leclercq, Jeanne (1976), Paradoxes du pouvoir local, Paris, Presses de la Fondation nationale des sciences politiques.

Blatrix, Cécile (1999), « La pratique politique de l'enquête publique », in CURAPP/CRAPS, La démocratie locale, Paris, Presses universitaires de France, p. 161-176.

Brassart-Goerg, Marie (1997), "Tel que je suis », Jean Oehler, Strasbourg, Éditions du Signe.

Bué, Nicolas ; Desage, Fabien, et Matejko, Laurent (2004), «La "métropole” n’est-elle qu'un mot ? Retour critique sur un "lieu commun" », in Dolez, Bernard et Paris, Didier, dir. (2004), Métropoles en construction. Territoires, politiques et processus, Paris, L'Harmattan, p. 71-93.

Courty, Guillaume, dir. (2005), Le travail de collaboration avec les élus, Paris, Michel Houdiard Éditeur. Delacroix, François (2007), Georges Frêche, la sagesse de la démesure, Céret, Alter ego éditions.

Desage, Fabien, et Sibille, Bastien (2011), « L'emprise de l'institué. Force et aléas du régime de "consensus" à la Communauté urbaine de Lille », in Lagroye, Jacques, et Offerlé, Michel, dir. (2011), Sociologie de l'institution, Paris, Belin, p. 151-175.

Dion, Stéphane (1986), La politisation des mairies, Paris, Economica.

Dulong, Delphine (1997), « Le président de la République : configuration et position prééminente », in Garrigou, Alain, et Lacroix, Bernard, dir. (1997), Norbert Élias, la politique et l'histoire, Paris, La Découverte.

Élias, Norbert (1985), La société de cour, Paris, Flammarion.

Garraud, Philippe (1989), Profession : homme politique. La carrière politique des maires urbains, Paris, L'Harmattan.

Gaxie, Daniel, et Lehingue, Patrick (dir.) (1984), Enjeux municipaux. La constitution des enjeux politiques dans une élection municipale, Paris, Presses universitaires de France et CURAPP.

Georgakakis, Didier (1995), «La double figure des conseils en communication politique », Sociétés contemporaines, $\mathrm{n}^{\circ} 24, \mathrm{p}$. 77-94.

George, Jocelyne (1989), Histoire des maires 1789-1939, Paris, Plon.

Haegel, Florence (1994), Un maire à Paris : mise en scène d'un nouveau rôle politique, Paris, Presses de Science Po.

Haroche, Claudine (1991), «Les cérémonies et les rituels de cour : des instruments d'une politique de communication », in CURAPP (1991), La communication politique, Paris, Presses universitaires de France, p. 183-195.

Hû, Grégory (2014), Les roses déracinées. Transformation du recrutement du personnel socialiste: des logiques sociales aux logiques politiques (fin XIX ${ }^{\text {ème }}$-2012), Thèse pour le doctorat de science politique, sous la direction d'Yves Déloye, Université de Strasbourg.

Jouandet, Jean (2013), Le mairisme ou la démocratie locale en trompe-l'œil. Reportage d'un Préfet aventuré dans le microcosme de Saint-Cyprien en Roussillon, Éditions Auteurs d'Aujourd'hui.

Juhem, Philippe (2006), « La production notabiliaire du militantisme au Parti socialiste », Revue française de science politique, vol. 56, $\mathrm{n}^{\circ}$ 6, p. 909-942.

Juhem, Philippe (2014), «Analyser les controverses politiques : le cas du débat sur l'identité nationale », in Husson-Rochcongar, Céline, et Jourdain, Laurence (2014), L'identité nationale : instruments et usages, Éditions du CURAPP. 
Lagroye, Jacques (1997), « On ne subit pas son rôle. Entretien avec Jacques Lagroye », Politix, $\mathrm{n}^{\circ} 38$, p. $7-17$.

Lascoumes, Pierre, dir. (2010), Favoritisme et corruption à la française. Petits arrangements avec la probité, Paris, Sciences Po Les Presses.

Le Bart, Christian (1992), La rhétorique du maire entrepreneur. Critique de la communication municipale, Paris, Pedone.

Le Bart, Christian (2003), Les maires. Sociologie d'un rôle, Villeneuve d'Ascq, Presses du Septentrion.

Lefebvre, Rémi (2004), «Le socialisme français soluble dans l'institution municipale ? Forme partisane et emprise institutionnelle : Roubaix (1892-1983) », Revue française de science politique, vol. $54, \mathrm{n}^{\circ} 2$, p. 237-260.

Lefebvre, Rémi (2007), « Qu'est le socialisme municipal devenu ? Politisation, dépolitisation, neutralisation de la question municipale au Parti socialiste (des années 1970 à nos jours) », in Arnaud, Lionel ; Le Bart, Christian, et Pasquier, Romain, dir. (2007), Idéologies et action publique territoriale, Rennes, Presses universitaires de Rennes, p. 51-79.

Legavre, Jean-Baptiste (1989), « Du militant à l'expert en communication politique », Politix, $\mathrm{n}^{\circ}$ 7-8, p. 80-90.

Legavre, Jean-Baptiste (1992), « Off the record. Mode d'emploi d'un instrument de coordination », Politix, $\mathrm{n}^{\circ} 19$, p. 135-157.

Lehingue, Patrick (1999), « Vocation, art, métier ou profession ? Codification et étiquetage des activités politiques », in Offerlé, Michel, dir. (1999), La profession politique XIX ${ }^{e}-X X^{e}$ siècles, Paris, Belin, p. 93-134.

Mabileau, Albert (1995), « De la monarchie municipale à la française », Pouvoirs, n 73, p. 7-17.

Madelin, Philippe (1997), Le Clan des chiraquiens, Paris, Seuil.

Maoudj, Karim (2007), Georges Frêche, grandes heures et décadence, Paris, Les éditions de Paris.

Marrel, Guillaume (2002), « Sociologie historique des carrières de cumul : une expérience prosopographique », in Déloye, Yves, et Voutat, Bernard, dir. (2002), Faire de la science politique. Pour une analyse sociohistorique du politique, Paris, Belin, p. 201-217.

Marrel, Guillaume, et Payre, Renaud (2001), « Le temps des mairies », Politix, n 53, p. 59-86.

Paoletti, Marion (2007), Décentraliser d'accord, démocratiser d'abord. Le gouvernement local en question, Paris, La Découverte.

Peraldi, Michel (2014), Ils ont volé la décentralisation! Pamphlet argumenté pour que la décentralisation soit rendue aux citoyens !, Paris, L'Harmattan.

Roubieu, Olivier (1999a), Des cadres gouvernants. Les hauts fonctionnaires des collectivités locales, Thèse pour le doctorat de science politique, sous la direction de Jacques Lagroye, Université Paris 1.

Roubieu, Olivier (1999b), « Des “managers” très politiques. Les secrétaires généraux des villes », in Dubois, Vincent, et Dulong, Delphine, dir. (1999), La question technocratique. De l'invention d'une figure aux transformations de l'action publique, Strasbourg, Presses universitaires de Strasbourg, p. 217-231.

Rowell, Jay (2006), Le totalitarisme au concret. Les politiques du logement en RDA, Paris, Économica.

Sawicki, Frédéric (1998), «La faiblesse du clientélisme partisan en France », in Briquet, Jean-Louis, et Sawicki, Frédéric, dir. (1998), Le clientélisme politique dans les sociétés contemporaines, Paris, Presses universitaires de France, p. 215-276. 
Smith, Andy, et Sorbets, Claude, dir. (2002), Le leadership politique et le territoire. Les cadres d'analyse en débat, Rennes, Presses universitaires de Rennes.

Sorbets, Claude (1983), « Est-il légitime de parler d'un présidentialisme municipal ? », Pouvoirs, $\mathrm{n}^{\circ} 24$, p. 115-116.

Troupel, Aurélia (2006), « Le retrait de délégation des adjoints : un vote du conseil purement formel », Pouvoirs locaux, ${ }^{\circ}$ 69, p. 35-40. 